\title{
A Study on the Optimization of Physical and Chemical Parameters for the Precipitate of Sodium Alkylsulfate with Cetylpyridinium Chloride
}

\author{
Sun Wha Oh, ${ }^{\dagger}$ Sung Doo Moon, Don Keun Lee, Dong Jae Lee, and Young Soo Kang ${ }^{\circ}$ \\ Department of Chemistm. Pukwong National Chiversitw, Busan 608-737. Korea \\ ${ }^{\dagger}$ Basic Science Research Institute, Puknong National Lhwersin, Busan 608-737, Korea \\ Received November 18,2003
}

\begin{abstract}
The optimum conditions for the most effective precipitate of surfactant complex of sodium alkylsulfate with cetylpyridinium chloride were studied in the aqueous solution. The parameters such as the alkyl chain length of anionic surfactants. molar ratio of two surfactants. temperature and the concentration of added $\mathrm{NaCl}$ in the aqueous solution were correlatively studied for the productivity of the precipitate formation. By the productivity. the optimum conditions to produce complex of anionic surfactant with cationic surfactant were the longer alkỵl chain, equivalent molar ratio between anionic and cationic surfactants. $0^{\circ} \mathrm{C}$ and $1.5 \mathrm{M} \mathrm{NaCl}$.
\end{abstract}

Key Words : Anionic surfactant. Cationic surfactant, Precipitate of surfactant complex

\section{Introduction}

Surfactants are the materials which have both the hydrophobic and hydrophilic groups in the same molecule. Surfactants exert various physical and chentical properties by their headgroups which have ionic or nonionic moiety. By the hydrophilic interaction of the headgroups with water. they result in a monolayer at the air/water interface. ${ }^{3}$ At a specific concentration, the monolayer can not form further more. and the surfactant molecules above critical micelle concentration (c.nt.c.) start to aggregate as micelles. This is called as c.m.c. So c.m.c. values are different by the kinds of surfactant and temperature. At c.m.c., the surface tension of water abruptly decreases according to the concentration of a surfactant, and there are also a sudden changes of solubilization, detergency. molar conductivity and osmotic pressure ${ }^{-5}$ The reason is that surfactants have an activity. the attraction with neighboring molecules should be minimum. The attraction in solution is expressed in termis of surface tension. So if the surface tension is small, surfactants will have surface activity and their diffusion will be easier. But it is limited by concentration factor: if the concentration increases to any limit below c.m.c., the above effect can be shown, but if the concentration is higher than c.m.c.. it is not shown. Because the surface tension reaches at saturation over c.m.c. ${ }^{2}$ In the concentration above c.m.c.. surfactants spontaneously fon spherical micelle in the aqueous solution. As the concentration increases higher than the concentration of spherical micelles. spherical micelles change to the rod-like and sheet-like micelle by surfactant species and temperature conditions. "The shape changes can be caused by the phase transition of the molecular assemblies of surfactants. This is possible by controlling the physical and chemical properties of surfactants during the phase transition. ${ }^{6} \mathrm{By}$ this way, it is possible to produce

\footnotetext{
Corresponding Author. Phone: +82-51-620-6379: Fax: +82-51-
} 628-8147; e-mail: y skang âpkúu. ac.ks precipitate of surfactant complex. The interaction between the hydrophilic group and the hydrophobic group can result in the precipitate and this is based upon modeling mainly from metal and surfactant or between different surfactants The model by metal and surfactant is to investigate the slope of solubility product $\left(\mathrm{K}_{\mathrm{sp}}\right)$ in c.m.c. It was found that the phase boundary of precipitate and the solid phase properties depended on the reactant concentration and $\mathrm{pH}$ of medium. ${ }^{6}$ So this model can be applied to the process that produces the colloidal heavy metal soaps in fatty acid solutions and the commercial surfactants. For the anionic and nonionic model, it is possible to expect the precipitate phase boundary. In this study. it is studied how the precipitate phase boundary can be obtained as the nonionic surfactant percentage increases, and the precipitate is reduced from $\mathrm{K}_{\mathrm{sp}}$ relationship between the anionic surfactant monomer and the total unassociated counterions. This model can be applied to the case that surfactants should be added to reduce the hard water property and oil recovery. The model by polyanion and polycation explains the various interactions involved in forming the polyelectrolyte complex through understanding those interactions. It serves some understanding of the basic characteristics by complexation in the biological system. That is to say. because of the complexation of biopolymers such as proteins. polysaccharides and nucleic acids. biofunctions-gene information. selective reactivity of enzymes and antigen-antibody reaction- are carried out. Precipitate of surfactants by anionic and cationic surfactant complexation in the aqueous solution can be evaluated by the usage of it in various fields. Precipitate is undesirable in cleaning of clothes, where simultaneous washing with fabric softening and antistatic incorporation in washing cycle that often requires the anionic and cationic surfactants both present. Conversely, the precipitate phenomena can be useful in a new process to improve the sweep efficiency in secondary oil recovery process where cationic and anionic reservoir separated by a brine spacer.

In spite of disadvantage of precipitate in surfactant solution. 
the precipitate enables the preparation of organic particle. which is possibly utilized in practical applications such as charge transfer complex and photodiode crystal detector materials. For the desirable precipitate, it is required that its optimum experimental conditions should be studied. There are many cases to analyze and research the results of the mixtures between the oppositely charged surfactants in the solution systems. ${ }^{6-8}$ The reason is that various phenomena from the mixtures and the interactions related to them are connected with many applications. Therefore, without the basic knowledge on the interactions, it will be impossible to understand the phenomena of the mixtures. In the previous study, it introduces the experimental method to expect the precipitate and the involved interactions-surface tension. foaming, electrophoretic mobility, solubility and so on. ${ }^{7}$

In this study, the organic surfactant particles have been prepared with cetylpyridinium chloride and sodium alkyl sulfate. The most efficient formation of organic surfactant particles was modified with hydrophobic alkyl chain length and molar ratio of two surfactant concentration, $\mathrm{pH}, \mathrm{NaCl}$ concentration and temperature.

\section{Experimental Section}

Materials. Sodium octylsulfate (SOS), sodium dodecylsulfate (SDS), sodium tetradecylsulfate (STS) and sodium dodecylbenzene sulfonate (SDBS) and cetylpyridinium chloride (CPCl) - were purchased from Aldrich Chemical

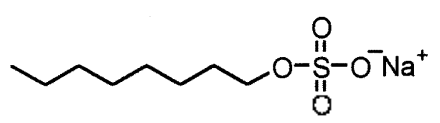

SOS (Sodium octylsulfate)

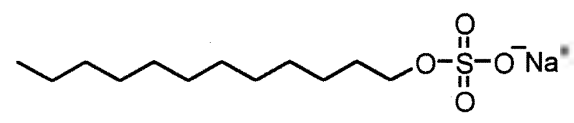

SDS (Sodium dodecylsulfate)

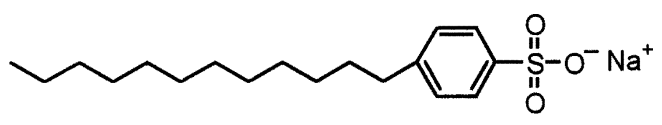

SDBS (Sodium dodecylbenzene sulfonate)

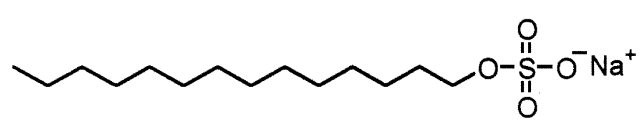

STS (Sodium tetradecylsulfate)

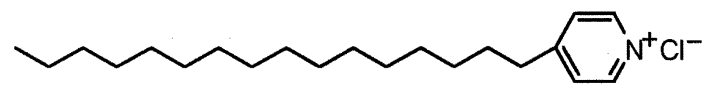

$\mathrm{CPCl}$ (Cetylpyridium chloride)

Figure 1. The structure of anionic and cationic surlactants.
Co. and used without any further purification. Their structures are shown in Figure 1.

Preparation of surfactant precipitates. A $0.288 \mathrm{~g}(0.00 \mathrm{l}$ mol) of SDS and $0.341 \mathrm{~g}(0.001 \mathrm{~mol})$ of $\mathrm{CPCl}$ were each dissolved in each $100 \mathrm{ml}$, of purified water to prepare $1 \mathrm{mM}$ solutions. Fach $10 \mathrm{ml}$. of $1 \mathrm{mM} \mathrm{SDS}$ and $1 \mathrm{mM} \mathrm{CPCl}$ solution was mixed with $10 \mathrm{~mL}$, of $1 \mathrm{mM} \mathrm{NaCl}$ solution at the same time. The resulting solution turned into turbid. All of the mixed solutions were prepared at the molar ratios of anionic alkyl sulfate to cationic alkylpyridinium chloride as $1: 1,1: 2$ and $1: 3$. The $\mathrm{pH}$ of the solutions was adjusted to $2,0,6.7,8.8$ and 12.0 by adding $0.1 \mathrm{M} \mathrm{HCl}$ and $0.1 \mathrm{M} \mathrm{NaOH}$ solutions. After the preparation of the mixed solutions, the solutions were cooled down to $6^{\circ} \mathrm{C}$ by putting in the refrigerator. To investigate the effect of temperature, the mixed solutions were stirred at $0,20,30$ and $55^{\circ} \mathrm{C}$ for 1 day, and then kept at room temperature for 4 days. The surfactant precipitates were isolated by the centrifugation at 10.000 rpm and dried under the reduced pressure. The precipitates of other anionic surfactant with CPCl were obtained and characterized as the same procedure. The weights of precipitates were determined by three times and average value was obtained. The precipitates of sodium alkylsulfate with $\mathrm{CPCl}$ with different molar ratios were spread in the pure water and the size distribution was checked with light scattering (Lexel-95 L aser Inc. Marverin 4700) using 488 nm argon laser in the aqueous solution at room temperature. Clemental analysis data were obtained with Flemental Analyzer (Elementar Analysensysteme, Flementar Vario Fl .) at $1150^{\circ} \mathrm{C}$.

\section{Results and Discussion}

Table 1 shows the results of elemental analysis on the composition of $\mathrm{N}, \mathrm{C}, \mathrm{S}$ and $\mathrm{H}$ of the precipitates produced by SDS and CPCI. The two surfactants were mixed by the molar ratios of $1: 1,1: 2$ and $1: 3$ of SDS to $\mathrm{CPCl}$. The composition ratio gained from all other different molar ratios did not show any difference of the composition of produced

Table 1. I: Iemental analysis data for dillerent molecular ralios of CPCl to $\mathrm{SDS}(\mathrm{pl}]=6.7 .0 .15 \mathrm{M} \mathrm{NaCl}$ and at room temperature)

\begin{tabular}{cccc}
\hline Ratio & Element & $\begin{array}{c}\text { txperimental } \\
\text { Composition }(\%)\end{array}$ & $\begin{array}{c}\text { Theoretical } \\
\text { Composition }(\%)\end{array}$ \\
\hline \multirow{3}{*}{$1: 1$} & $\mathrm{C}$ & 2.69 & \\
& $\mathrm{C}$ & 67.19 & \\
& $\mathrm{~S}$ & 5.80 & \\
$1 \mathrm{H}$ & 10.60 & \\
$1: 2$ & $\mathrm{C}$ & 2.49 & $\mathrm{~N}: 2.00$ \\
& $\mathrm{C}$ & 68.24 & $\mathrm{C}: 70.00$ \\
& $\mathrm{~S}$ & 5.84 & $\mathrm{~S}: 6.00$ \\
& $\mathrm{H}$ & 10.79 & $11: 11.00$ \\
$1: 3$ & $\mathrm{~N}$ & 2.65 & \\
& $\mathrm{C}$ & 69.09 & \\
& $\mathrm{~S}$ & 5.66 & \\
\hline & $\mathrm{H}$ & 10.88 & \\
\hline
\end{tabular}




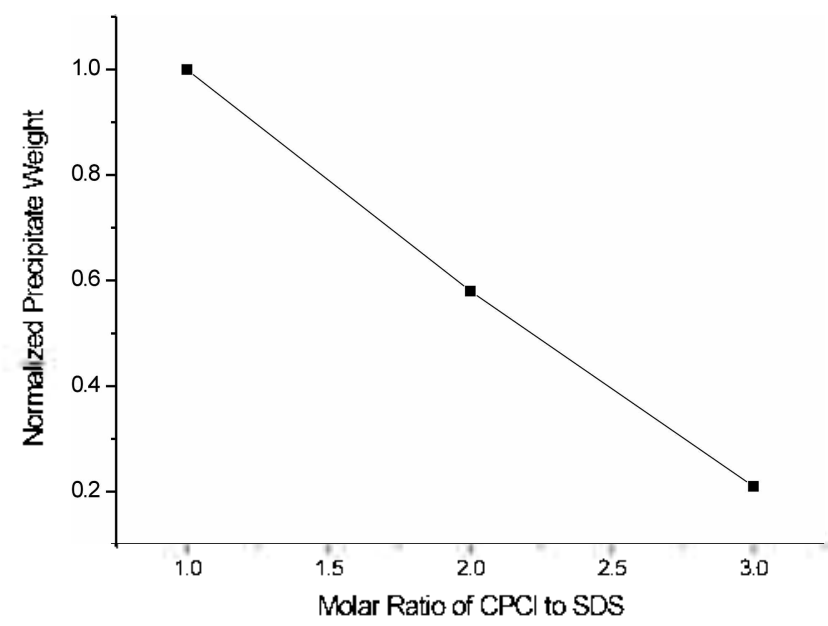

Figure 2. The weight amount of surfactant precipitales prepared by diljerent molar ratios of SI)S to CPCl $(\mathrm{pll}=6.7 .0 .15 \mathrm{M} \mathrm{NaCl}$. room temperature).

precipitates among them. Anionic and cationic surfactants are represented as $\mathrm{AS}^{-}$and $\mathrm{CS}^{-}$, respectively. This indicates that the oppositely charged surfactants form the precipitates by ecjui-molar ratio as

$$
\mathrm{AS}^{-}(\mathrm{aq})+\mathrm{CS}^{\prime}(\mathrm{aq})^{-} \operatorname{ASCS}(\mathrm{s})
$$

The efficiency of surfactant precipitate formation was determined by weighing the precipitates. Figure 2 shows the weight of surfactant precipitates prepared by different molar ratios of SDS to CPCl in the $0.15 \mathrm{M}$ acjueous $\mathrm{NaCl}$ solution of $\mathrm{pH}=6.7$ at room temperature. $\mathrm{Y}$-axis is represented with Normalized Precipitate Weight which is the ratio of each precipitate weight to maximum precipitate weight. The weight of the precipitates was the highest at the equi-molar ratio between SDS and CPCl. The mean sizes of precipitates with $1: 1,1: 2$ and $1: 3$ molar ratio of SDS/CPCl with light scattering were determined as 439,326 and $308 \mathrm{~nm}$, respectively. These indicate that the weight and size of precipitates were critically affected by the molar ratio. Even with different amount of precipitates, the composition of precipitates was not changed by the molar ratios. This can be explained as the basic equilibrium among monomers. precipitates and micelles can be oriented to precipitates by the equi-molar ratio between $\mathrm{SDS}$ and $\mathrm{CP} C \mathrm{Cl}$. The schematic drawing on the basic equilibria among micelle. monomer and precipitate are shown in rigure 3 . The precipitate phase boundary of SDS and $\mathrm{CP}^{\prime} \mathrm{Cl}$ with different molar ratio for the efficient formation of precipitates can be drawn as rigure 4 . When monomers are aggregated, they are destined to form micelles or precipitates. At this point, the factor to decide their formation is the concentration of two oppositely charged surfactants. Micelles are formed at the concentration above their c.m.c. of the surfactants. On the other hand, precipitates will be formed if the concentration product of two surfactants is larger than the solubility product. Therefore, if the molar ratio of $\mathrm{CS}^{-} / \mathrm{AS}^{-}$is large, the equilibria of micelle-monomer and monomer-precipitate will be established. For this case, all parts, such as monomer.

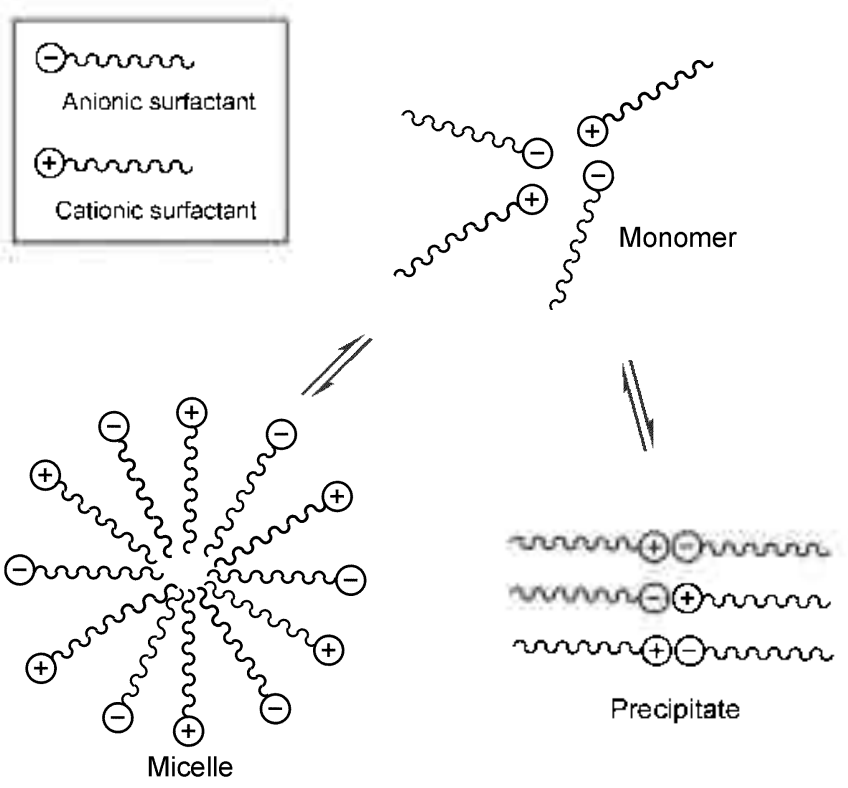

Figure 3. The schematic drawing on basic equilibria among miselle. monomer and precipitate.

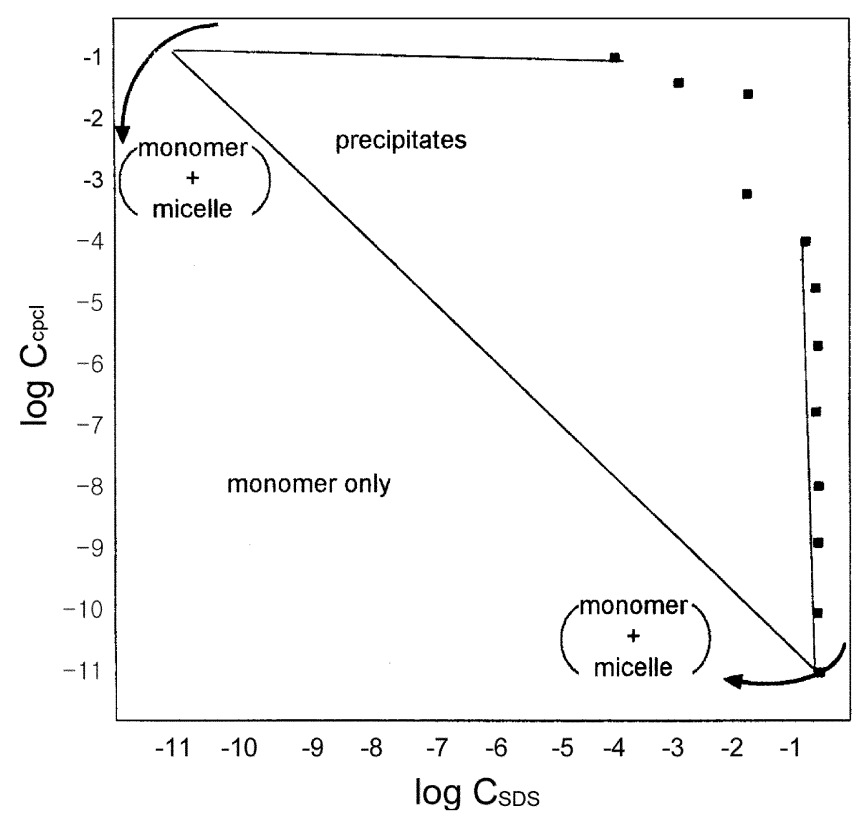

Figure 4. The precipitate phase boundary of SDS/CPCl complexes (pH 6.7.0.15 M VaCl. room temperature).

micelle and precipitate, are included in the precipitate phase boundary. But, considering the precipitate phase boundary of the current experiment, the line of the equi-molar ratio only passes across the region from monomer to precipitate. This shows that the equilibrium between monomer and precipitate will be dominant in the solution of the surfactant complex. Therefore, the most effective precipitate in the equi-molar ratio can be understood by the equilibriums. Figure 5 and 6 show that the effects of $\mathrm{pH}$ and temperature of solution on the precipitate of surfactant mixtures. The larger amount of precipitates was formed in the higher $\mathrm{pH}$ and the lower temperature. To increase $\mathrm{pH}$ of solution, more 


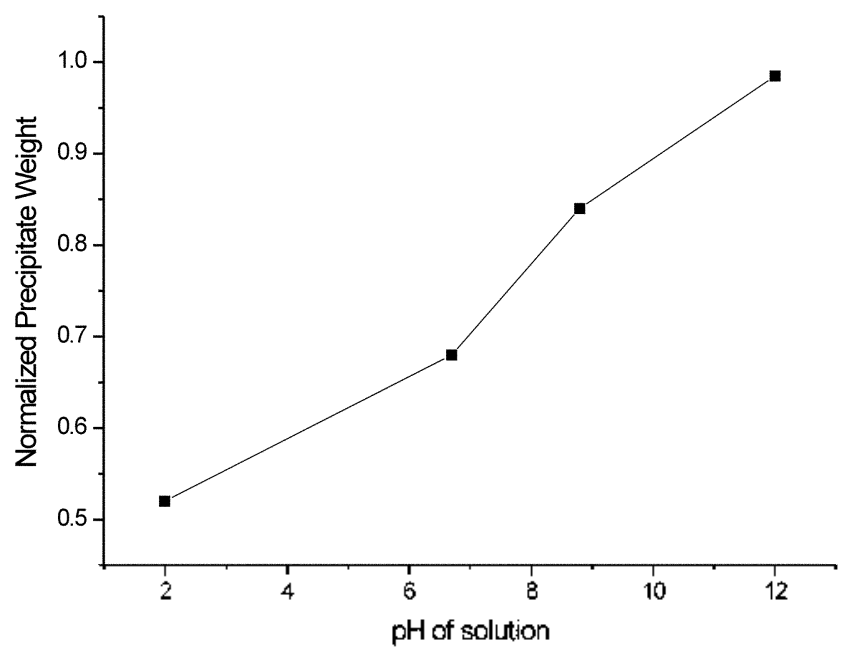

Figure 5. The pI I effect on the precipitate of SDSCPCI complexes.

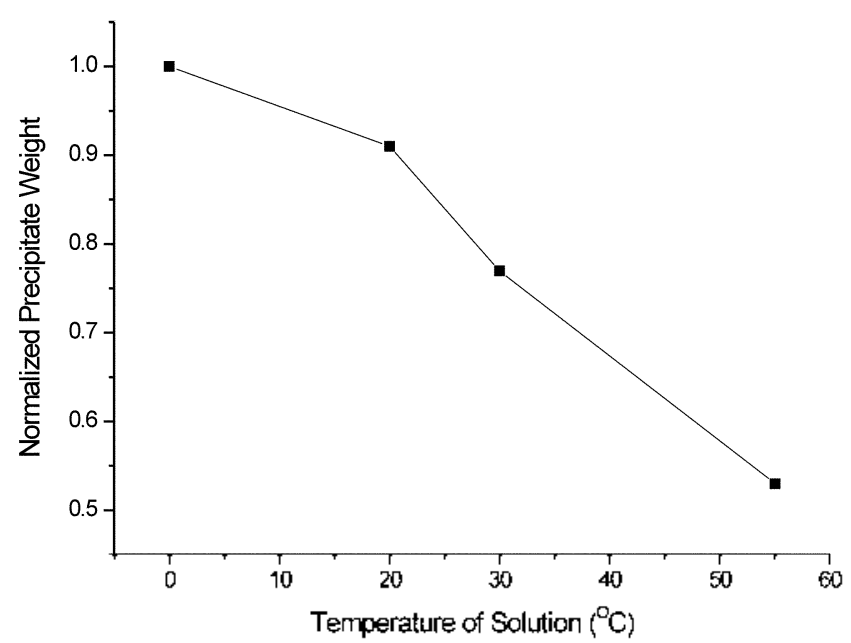

Figure 6. The temperature ellect on the precipitate of SDS/C.PCI complexes.

$\mathrm{NaOH}$ solution was added into the mixed solution of surfactant solution. The larger amount of precipitates in the higher $\mathrm{pH}$ of solution is possibly caused by the decreased surface charge potential of the micelles by added $\mathrm{NaCl}$ into the surfactant solution. The added $\mathrm{Na}^{+}$and $\mathrm{Cl}^{-}$ions can decrease the interface charge of micelles working as counter ions to the interface. This results in the less formation of micelles and the equilibrium between monomer-precipitate tends to be dominant in the solution. The less formation of micelle in higher $\mathrm{pH}$ of the solution in the presence of more $\mathrm{NaCl}$ is already reported by Graham. ${ }^{8}$ 'This is schematically shown in Figure 7. When $\mathrm{NaCl}$ is added the size of the precipitate of mixed micelle is increased due to the lowered c.m.c. by $\mathrm{NaCl}$. This leads to the equilibrium of monomerprecipitate to be dominant. The more effective formation of precipitates in the lower temperature is shown in Figure 6 . Ihis is attributed to the lower solubility product of the surfactant precipitate in the lower temperature. This lets the equilibrium of monomer-micelle move toward that of monomer-precipitate. In the solution, in addition to the diffusion by the surfactant molecules themselves, there is the

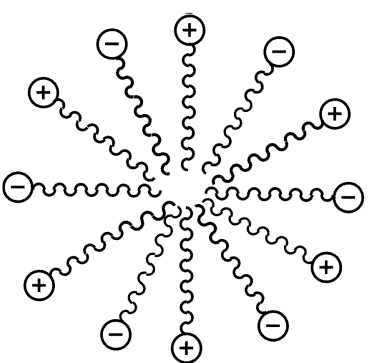

(a)

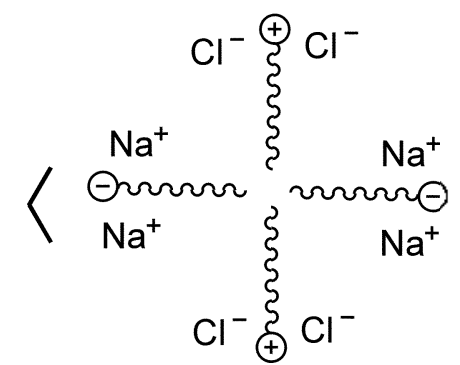

(b)
Figure 7. Schematic drawing lo compare the size between (a) usual mixed micelle and (b) the mixed micelle screened by $\mathrm{NaCl}$.

mass transportation by the fluid mass, which is termed as convection current." This convection current can be controlled by phase, temperature and stirring. If the temperature of solution become homogeneous in the whole solution, the convection current is kept, but the latter is higher, the current is directed to dissolve the precipitates into the solution. ${ }^{10}$ In this system, the temperature of the solution containing the precipitate is $6^{\circ} \mathrm{C}$. Therefore, the more effective precipitate is possible in the lower temperature.

The effect of $\mathrm{NaCl}$ concentration on the surfactant precipitate is shown in Figure 8 . The weight of precipitates increases with increasing concentration of $\mathrm{NaCl}$ from $0 \mathrm{M}$ to $1.5 \mathrm{M}$ and then reaches a plateau. The counter ions play a role of contracting charges of the surfactant headgroups, which results in the decrease of the repulsion by the electrostatic interaction between the headgroups, which leads to repel the micelle formation. Of course, the two anionic and cationic surfactants will not have electrostatic repulsion. But, in the case of their concentration less than c.m.c., the surface potential rather than the adsorption will influence with the bonding. Therefore, by the higher concentration of $\mathrm{NaCl}$, the precipitate will be formed easily due to the decreased c.m.c. of surfactant micelles. This results in the lower aggregation number of the micelle and

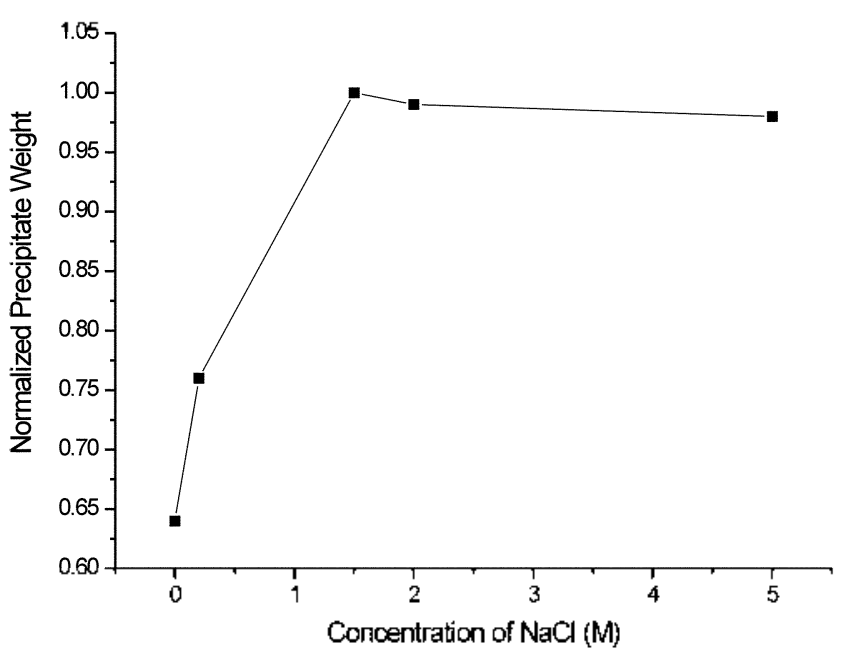

Figure 8. The eflect of $\mathrm{NaCl}$ concentration on the precipitate of SDS CPCI complexes. 


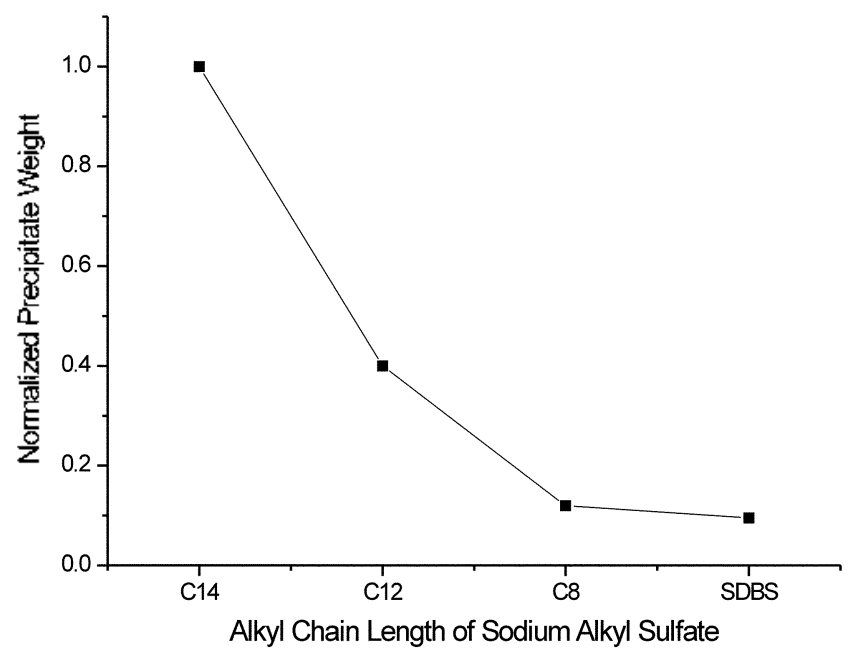

Figure 9. The alkyl chain lenglh elfect of sodium alkylsultate on the precipitates with CPCI. (C8: SOS. C12: SDS. C14: SIS).

makes the equilibrium be transferred to the precipitates in the higher $\mathrm{NaCl}$ concentration.

The alkyl chain length effect of sodium alkyl sulfate on the precipitates with $\mathrm{CPCl}$ is shown in Figure 9. The weight of precipitates was decreased with decreasing alkyl chain length from tetradecyl to octyl chain length and reached the least amount by the dodecylbenzene sulfate. This can be explained by two factors. The first one is the decreasing hydrophobicity of the precipitate with decreasing alkyl chair length of the surfactant. When anionic surfactant with longer alky] chain forms precipitates with cationic surfactants, $\mathrm{CPCl}$, the precipitates formed by two surfactants will have more hydrophobicity than the pure surfactants. This will make the precipitates diffuse easily and increase their adsorption onto the water surface. ${ }^{2}$ As a result. c.m.c. of the mixed micelle will be lowered, the equilibrium of monomerprecipitate will be dominant in the solution. The second one is the aromatic hydrocarbon moiety of compounds. The $\pi$ electron in the aromatic ring of dodecylbenzene sulfate will have a partial hydrogen bonding with water molecules." Ihis probably increases the solubility of the precipitates and results in the lowest amount of precipitate of dodecylsulfate with $\mathrm{CPCl}$.

\section{Conclusions}

Precipitates of alkylsulfate with cetylpyridinium chloride were successfully prepared in the aqueous solution. The optimized conditions for the most efficient formation of precipitates were obtained as longer alkyl chain length of alkylsulfate, higher $\mathrm{pH}$ and $1.5 \mathrm{M} \mathrm{NaCl}$. The largest weight of precipitates was obtained with the equi-molar ratio between anionic and cationic surfactants.

Acknowledgement. This work was supported by Pukyong Research Fund in 2002, Korea Research Foundation Grant (KRF-2002-050-C00007) and the Brain Busan 21 Project in 2004.

\section{References}

1. Fujinoto. T. Aew Introduction to Surface Active Agents: Sunyo Chemical Industrics Ltd.: Tokyo. 1996.

2. Shaw. D. I. Introdtetion to Colloid and Sinfoce Chemistry. 2nd Fd.: Butterworths: london, 1966.

3. Bennet1. D. I:.: (jallard, 13. S.: Abot. N. 1.. J. Am. (hem. Soc. 1996. 118.499

4. (a) Gallardo. B. S.: Hła. M. I.: Atolt. N. L. Langmir 1995. //. 4209. (b) Kin. H.: Lim. K. Bull. Korem Chem. Soc. 2003. 2t. 1449. (c) Lec. B. J. Korem Chem. Soc. 1998. 42. 519.

5. Tanford. C. The Hydrophobic Fffect: Fomation of Wicelles and Biological Wembranes: John Wiley \& Sons: Vew York. 1979.

6. Amante, I. C.: Scamehom, J. J.: Harwell. J. I. .) C'olloid and Inerface Science 1991. 144. 243 .

7. Goddard. E. D.: Hanmans. R. B. J. Collod and Interfoce Science 1976. 55.73

8. Stellner. K. I..: Amante, J. C.: Scamehorn. J. F.: I Iarwell, J. II. J. colloid and hiterface science 1988. /23. 186.

9. Adamson. A. W. Phwical (hemisty of Sufaces. 517 Jid. John Wiley \& Sons. Ine: New York. 1990.

10. Hoycr. H. W.: Marmo. A.: Zollner. M. Colloid and herfoce Science 1984. 119.1221.

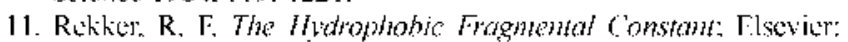
New York. 1977. 